\title{
Early Abdominal Ectopic Pregnancy Masquerading as a Missed Miscarriage
}

\author{
Joy Qing En Wonga, Yu Hui Limª, b
}

\begin{abstract}
A 43-year-old lady, gravida 2 para 0, presented to our emergency department with complaints of vaginal bleeding and lower abdominal pain. Her urine pregnancy test was positive. She was unable to recall her last menstrual period. A trans-vaginal ultrasound revealed a pregnancy with a crown rump length of $47 \mathrm{~mm}$ corresponding to 11.4 weeks with no fetal heartbeat detected. She was diagnosed with a missed miscarriage and was sent for a second confirmatory scan. The repeat scan was concordant with the initial scan and she was counseled for an evacuation of uterus. Her serum beta human chorionic gonadotropin level was 45,195 IU/L and her hemoglobin level was $6.5 \mathrm{~g} / \mathrm{dL}$. She underwent an evacuation of uterus as planned, but the Hegar dilator was only able to be advanced to a cavity length of $6 \mathrm{~cm}$ with minimal products of conception obtained. A bedside ultrasound was performed and it showed that the Hegar dilator was in the uterine cavity but not in continuity with the gestational sac and fetus. The diagnosis of an ectopic pregnancy was made and the surgery was converted to a diagnostic laparoscopy. On entry into the abdominal cavity, there was frank hemoperitoneum with adhesions limiting access to the pelvis, therefore decision was made to convert to laparotomy. The findings at laparotomy revealed a large inflamed left tubo-ovarian complex with tubal rupture and expulsion of the entire fetus and placenta into the Pouch of Douglas (POD). The diagnosis of a secondary implantation of the ectopic pregnancy in the POD after tubal rupture was confirmed and we performed adhesiolysis and left salpingectomy. The patient recovered uneventfully and the final histology showed products of conception within the lumen of the left fallopian tube in keeping with ruptured tubal ectopic pregnancy.
\end{abstract}

Keywords: Abdominal pregnancy; Ectopic pregnancy; Missed miscarriage

\section{Introduction}

Abdominal pregnancy is a rare form of ectopic gestation. It is

Manuscript submitted April 30, 2020, accepted May 7, 2020

Published online May 28, 2020

aDivision of Obstetrics and Gynaecology, KK Women's and Children's Hospital, Singhealth, Singapore

${ }^{b}$ Corresponding Author: Yu Hui Lim, Division of Obstetrics and Gynaecology, KK Women's and Children's Hospital, 100 Bukit Timah Road, 229899, Singapore. Email: lim.yu.hui@singhealth.com.sg

doi: https://doi.org/10.14740/jmc3484 commonly misdiagnosed as other early pregnancy complications owing to its vague presenting signs and symptoms, which clinicians may overlook. Ultrasound as the investigation of choice has low rates of diagnostic accuracy, and findings can be misleading. We describe a case of an 11-week abdominal pregnancy, which was initially misdiagnosed as a miscarriage as the pregnancy appeared to be intrauterine on sonography. During evacuation of the uterus, a repeat ultrasound with an intrauterine dilator helped to delineate the empty uterus and led to revision of the diagnosis. The patient underwent laparotomy, left salpingectomy and successful removal of the abdominal pregnancy and placenta. In order to make an early diagnosis of abdominal pregnancy, it is necessary to take into account the complete clinical scenario and perform detailed sonographic examination. Importantly, the clinician must have a high index of suspicion for this unusual condition.

Abdominal ectopic pregnancy is a rare but dangerous condition. It is defined as a pregnancy that occurs in the abdominal cavity outside of the female reproductive organs. Early abdominal pregnancies (EAPs) occur at or before 20 weeks of gestation [1]. Incidence varies widely based on factors such as socio-economic status, geography and antenatal attendance [1]. In one large study, abdominal ectopic pregnancies were found to constitute approximately $1 \%$ of all ectopic pregnancies, occurring in 10.9 per 100,000 live births [1]. Mortality rates are 7.7 times higher than in tubal pregnancies, and 89.8 times higher than in intrauterine pregnancies [1]. The top three sites of EAPs are pouches around the uterus (24.3\%), serosal surface of the uterus and tubes (23.9\%), and multiple abdominal organs (12.8\%) [2]. Ultrasound is currently considered to be the investigation of choice to facilitate the diagnosis of extra-uterine pregnancy, with Allibone et al providing guidelines for the diagnosis of abdominal pregnancy [3]. Despite this, the reported diagnostic errors in different series have ranged from $50 \%$ to $90 \%$ [4-6], and diagnosis is more often made intraoperatively $[4,5]$. With this case, we hope to demonstrate the importance of a high index of suspicion for abdominal pregnancies and also the need to correlate clinical examination findings with laboratory and radiological investigations in order to come to an accurate diagnosis for early pregnancy complications.

\section{Case Report}

A 42-year-old lady, gravida 2 para 0 , presented to our emergency department with complaints of vaginal bleeding and 

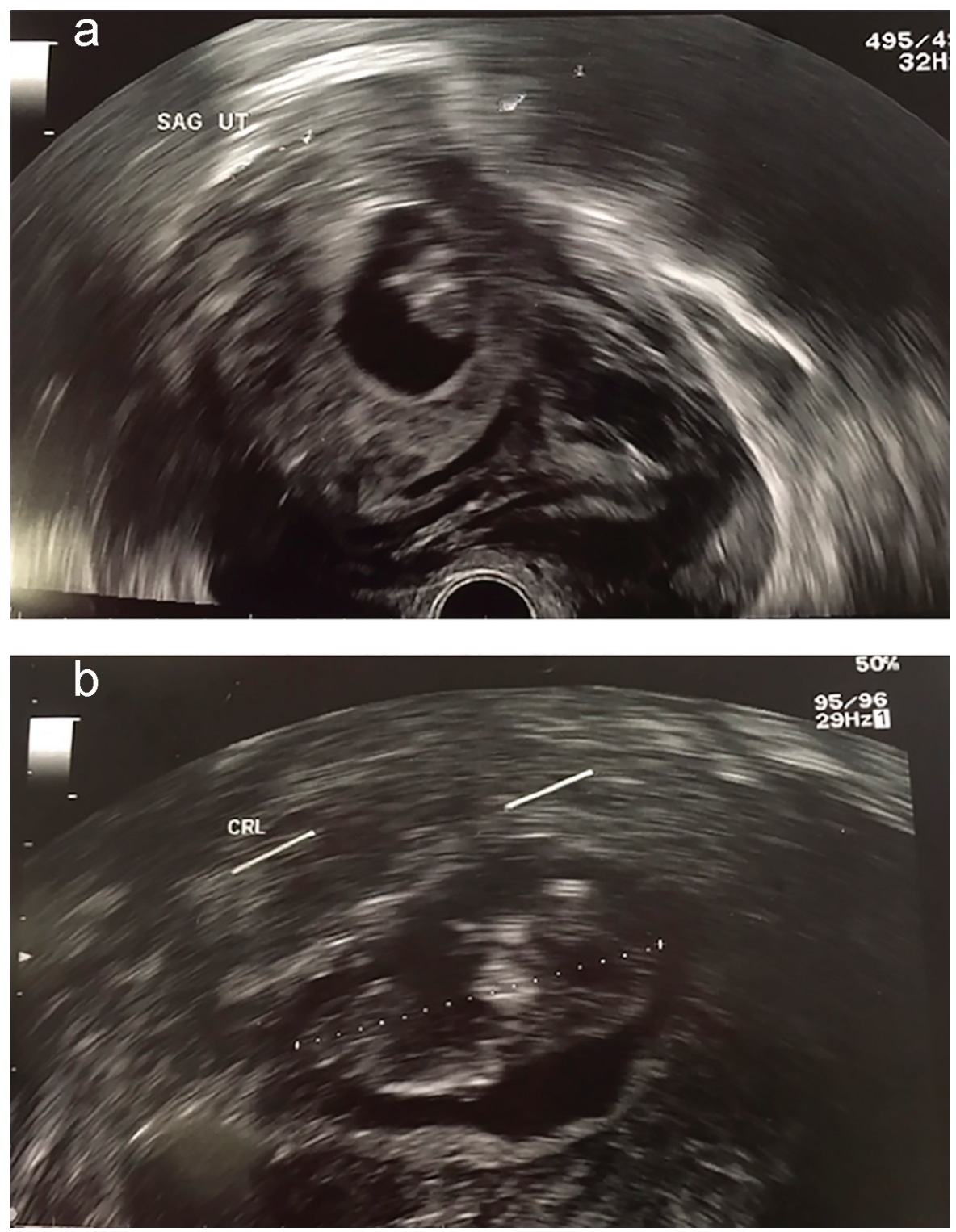

Figure 1. (a) Sagittal view of uterus. (b) Measurement of crown rump length (CRL).

lower abdominal pain for 1 day, with a positive urine pregnancy test. She had no past medical history, and surgical history comprised of one previous termination of pregnancy. She was unable to recall her last menstrual period but reported taking abortion pills 2 weeks ago. Physical examination revealed an enlarged uterus of 12 weeks size, with mild tenderness on palpation of the suprapubic region but no rebound or guarding. Speculum examination revealed a closed cervix with minimal vaginal bleeding.

Bedside trans-vaginal ultrasound revealed a pregnancy with a crown rump length of $47 \mathrm{~mm}$ corresponding to 11.4 weeks, with no fetal heartbeat detected. She was provisionally diagnosed with a missed miscarriage and underwent a second scan for confirmation. The repeat scan by a different operator was concordant with the initial scan (Fig. 1a, b). The patient was counselled on the diagnosis accordingly and consented to a surgical evacuation of the uterus. Laboratory investigations revealed a serum beta human chorionic gonadotropin (bhCG) level of 45,195 IU/L and hemoglobin level of $6.5 \mathrm{~g} /$ $\mathrm{dL}$, prompting initiation of a packed cell transfusion. At that time, her anemia was attributed to vaginal loss from the miscarriage and physiological anemia from the pregnancy. Her vital signs remained stable.

Taking into consideration the history, clinical examination and ultrasound findings, our working diagnosis was that of a missed miscarriage. Another possible differential was an ectopic pregnancy, more commonly tubal in origin. However, there was a low clinical suspicion for this condition in view of the fact that the fetus was entirely visualized in its gestational sac as an intrauterine pregnancy, confirmed on two ultrasounds done by different operators. Clinical examination of the patient also revealed a soft abdomen with only mild tenderness in the 


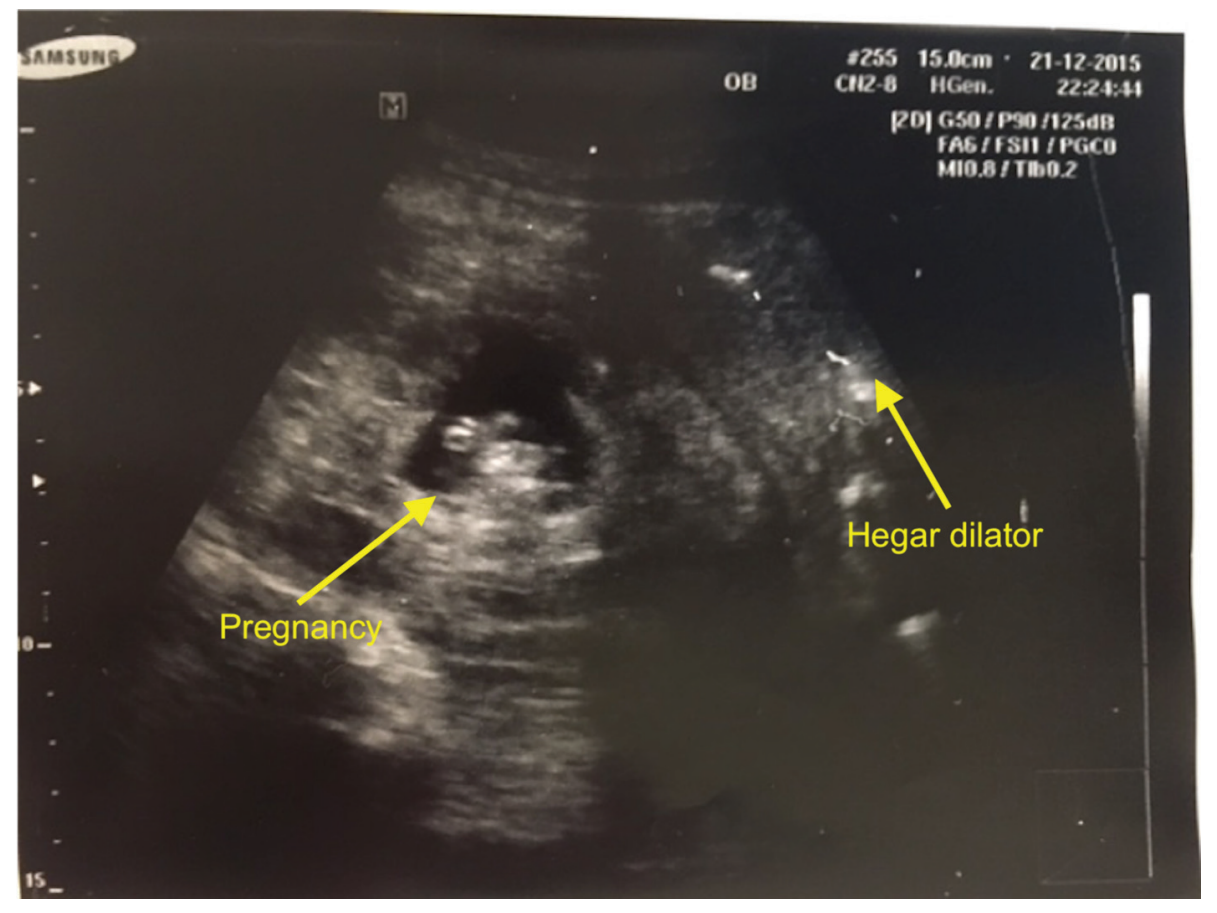

Figure 2. Intra-operative finding of abdominal pregnancy.

suprapubic region and no overt signs of peritonism.

The patient underwent evacuation of the uterus under general anesthesia as planned. A repeat vaginal examination was done intra-operatively and there was an unusual finding of a mass felt in the Pouch of Douglas (POD). A Hegar dilator was used to gently dilate the cervical os, but it was only able to be advanced to a cavity length of $6 \mathrm{~cm}$. Minimal products of conception were obtained. She was quickly reassessed with an intra-operative ultrasound. This time, the ultrasound clearly demonstrated that the Hegar dilator was in the uterine cavity, but it was not in continuity with the gestational sac and fetus (Fig. 2). This prompted revision of the diagnosis to an abdominal ectopic pregnancy and we proceeded to perform a diagnostic laparoscopy.

Upon entering the abdominal cavity there was frank hemoperitoneum with adhesions, limiting access to and visualization of the pelvis. Therefore, the decision was made to convert the operation to an exploratory laparotomy. This revealed a large and inflamed left tubo-ovarian complex with inflamed omentum covering the uterine surface. The entire fetus and placenta had been expelled into the POD. Blood clots were evacuated and the origin of the ectopic was identified to be the fimbrial end of the left tube, which was actively bleeding. The uterus was otherwise normal. We performed adhesiolysis and left salpingectomy, and fetus and placenta were removed in its entirety (Fig. 3). The total estimated blood loss was $1.8 \mathrm{~L}$.

The patient recovered uneventfully after surgery and was discharged on the second postoperative day. Histology showed products of conception within the lumen of the left fallopian tube in keeping with a ruptured tubal ectopic pregnancy. Final diagnosis was that of a secondary abdominal ectopic preg- nancy, with implantation in the POD after tubal rupture. The patient was given an outpatient follow-up appointment. However, she defaulted on the appointment as she had made plans to return to her home country.

\section{Discussion}

We describe a case of misdiagnosis of an EAP masquerading as a missed miscarriage. This highlights the clinical challenge in differentiating abdominal ectopic pregnancies from intrauterine pregnancies in early gestation.

In terms of initial presentation, there appears little that could have raised our suspicion for an abdominal pregnancy. The patient did not have any risk factors for abdominal preg-

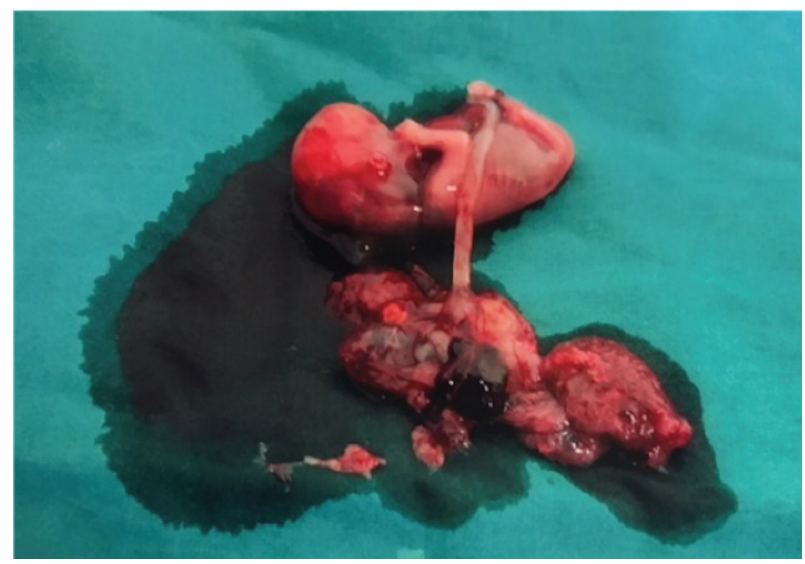

Figure 3. Fetus and placenta 
nancy such as previous ectopic pregnancies or tubal surgery, endometriosis, history of pelvic inflammatory disease or current use of an intrauterine device [4]. Furthermore, there are no known pathognomonic signs and symptoms of abdominal pregnancy [4]. Her symptoms of abdominal pain and vaginal bleeding as well as the findings of abdominal tenderness and a closed cervix, although in keeping with the diagnosis of an abdominal pregnancy $[3,6,7]$, are commonly seen in threatened or missed miscarriages. Gastrointestinal symptoms such as severe constipation, which are commonly present in patients with abdominal pregnancy [3], were not present in this patient. Other signs of abdominal pregnancy such as a radically displaced anterior, superior and uneffaced cervix, palpation of the uterus as a mass distinct from the pregnancy, easily palpable fetal parts or very difficult palpation of the fetus and abnormal fetal lie [6], would have been difficult to appreciate at such an early gestation.

Upon retrospective analysis of this case, one could highlight that a low hemoglobin level of $6.5 \mathrm{~g} / \mathrm{dL}$ in a previously well patient with no past history of underlying anemia should have prompted further investigation. In one case series of EAP, 10/15 patients had anemia with hematocrit $<30 \%$ [7]. Although anemia is a common feature in abdominal pregnancy $[3,6]$, it is not an uncommon finding in early pregnancy. Moreover, the patient was clinically stable. Nonetheless, taking into consideration the clinical scenario, other sources of bleeding ought to have been considered. Importantly, a ruptured ectopic pregnancy would have been important differential to exclude.

Ultrasound is the tool of choice for diagnosis of EAP [3-6]. Unfortunately, as aforementioned, its yield is low with approximately $50 \%$ accuracy when used along with clinical evaluation [4], and the diagnosis is instead frequently made intra-operatively during diagnostic laparoscopy or laparotomy $[4,5]$. The main problem is that neither clinicians nor ultrasonographers readily bear in mind the possibility of this rare diagnosis $[6,7]$. This holds true in our case report whereby two independent operators each performed an ultrasound scan for the patient but did not identify features consistent with an abdominal ectopic pregnancy. In a case series of 20 proven abdominal pregnancies, it was found that the retrospective diagnosis could be made sonographically in $90 \%$ of the cases, although it was initially missed in $25 \%$ of the cases in the hands of experienced obstetricians and sonographers[7].

On top of ultrasound criteria previously described by Allibone et al [3], Gerli et al proposed a new set of criteria whereby diagnosis of abdominal pregnancy in the first trimester required: 1) Absence of an intrauterine gestational sac; 2) No evidence of tubal dilatation or a complex adnexal mass; 3 ) A gestational sac surrounded by loops of bowel and separated from the uterus; and 4) Free mobility of the gestational sac [8]. However, in this case, the gestational sac was thought to be intrauterine and it was neither surrounded by bowel nor freely mobile, therefore contributing to the misdiagnosis at the initial evaluation. Additionally, in most cases of sonographically diagnosed abdominal pregnancies, the gestation sac was found cephalad to the uterus with downward displacement or compression of the uterus and bladder [9]. This was not the case in our patient, where the gestational sac in the POD was mistaken for an intrauterine pregnancy in a retroverted uterus.

The most frequent and reliable finding in sonographs of abdominal pregnancies is the identification of the empty uterus as separate from the fetus [9]. In light of this, the radiographer should strive to clearly delineate the uterine cavity in its entirety from uterine fundus to cervix on the sagittal view, in order to prevent misdiagnosis. Importantly, both trans-abdominal as well as trans-vaginal scans should be undertaken in all patients with early pregnancies in order to obtain reliable transverse and sagittal images.

In this case, the diagnosis was clinched during examination under anesthesia, with the aid of an intra-operative ultrasound and Hegar dilator to demarcate the intrauterine space as separate from the fetus (Fig. 2). Others have proposed the trans-cervical placement of a Foley's catheter with inflation of its balloon to demonstrate the empty uterus on ultrasound [10]. This may be helpful when there is a clinical suspicion for abdominal pregnancy with difficulty in distinguishing the intra-abdominal compartments. In our patient, misdiagnosis was what first prevented initiation of the appropriate management. Once we arrived at the diagnosis of an abdominal pregnancy, emergency surgical treatment was carried out smoothly and the patient recovered well with no postoperative complications.

Besides enabling more rapid institution of management, early diagnosis of abdominal pregnancy would also allow for alternative treatment methods which could lower rates of associated maternal morbidity and mortality, and give rise to better fertility outcomes $[4,5]$. Primary surgical management is performed in $87 \%$ of cases, being more frequently opted for because many of the patients presented initially with intra-abdominal bleeding [2]. Identification of the abdominal ectopic pregnancy prior to bleeding would aid in preoperative planning and could allow a less invasive operation [4, 8]. EAPs are also more easily managed with laparoscopy as compared to later in gestation [8]. This is especially because when diagnosed relatively late, the placenta and its area of attachment are larger with broader and deeper invasion of the villi, and this has greater potential to cause life-threatening bleeding in the event of separation [4]. To prevent bleeding, the placenta is sometimes left in situ, resulting in higher morbidity and risk of requiring a second operation [5]. In this patient, the placenta was completely removed because it was easily accessible, relatively small in size, and was not attached to a vital organ. More recently, early diagnosis has also led to primary medical treatment with potassium chloride or methotrexate as alternatives to surgery $[2,4]$.

\section{Conclusion}

This case illustrates the difficulty in making an accurate diagnosis of EAP, especially when presenting symptoms tend to be vague and common in the spectrum of early pregnancy complications. Ultrasound is used as an adjunct to clinical examination and evaluation of the patient. However, it cannot be overemphasized that the diagnosis of an abdominal ectopic pregnancy can still be missed, especially if clinician has a low index of suspicion for the condition. Early diagnosis would 
give rise to better maternal outcomes and to this end, we urge clinicians to bear in mind this uncommon but important differential.

\section{Acknowledgments}

None to declare.

\section{Financial Disclosure}

None to declare.

\section{Conflict of Interest}

None to declare.

\section{Informed Consent}

Patient consented for her information and images to be used for publication in this case report.

\section{Author Contributions}

JQE Wong and YH Lim: conceptualization; JQE Wong: writing (original draft preparation); JQE Wong and YH Lim: writing (review and editing).

Date AvailabilityThe authors declare that data supporting the findings of this study are available within the article.

\section{References}

1. Atrash HK, Friede A, Hogue CJ. Abdominal pregnancy in the United States: frequency and maternal mortality. Obstet Gynecol. 1987;69(3 Pt 1):333-337.

2. Poole A, Haas D, Magann EF. Early abdominal ectopic pregnancies: a systematic review of the literature. Gynecol Obstet Invest. 2012;74(4):249-260.

3. Allibone GW, Fagan CJ, Porter SC. The sonographic features of intra-abdominal pregnancy. J Clin Ultrasound. 1981;9(7):383-387.

4. Agarwal N, Odejinmi F. Early abdominal ectopic pregnancy: challenges, update and review of current management. The Obstetrician \& Gynaecologist. 2014;16:193198.

5. Shaw SW, Hsu JJ, Chueh HY, Han CM, Chen FC, Chang YL, Chao AS, et al. Management of primary abdominal pregnancy: twelve years of experience in a medical centre. Acta Obstet Gynecol Scand. 2007;86(9):1058-1062.

6. Martin JN, Jr., McCaul JFt. Emergent management of abdominal pregnancy. Clin Obstet Gynecol. 1990;33(3):438447.

7. Martin JN, Sessums JK, Martin RW, Rick W, Pryor JA, Morrison JC. Abdominal Pregnancy. Obstetrical \& Gynecological Survey. 1988;43:540-541.

8. Gerli S, Rossetti D, Baiocchi G, Clerici G, Unfer V, Di Renzo GC. Early ultrasonographic diagnosis and laparoscopic treatment of abdominal pregnancy. Eur J Obstet Gynecol Reprod Biol. 2004;113(1):103-105.

9. Graham D, Johnson TR, Sanders RC. Sonographic findings in abdominal pregnancy. Journal of Ultrasound in Medicine 1982;1:71-74.

10. Ekele BA, Ahmed Y, Nnadi D, Ishaku K. Abdominal pregnancy: ultrasound diagnosis aided by the balloon of a Foley catheter. Acta Obstet Gynecol Scand. 2005;84(7):701-702. 